\title{
On the Development of an Open-Source Learning Management System for Engineering Students
}

\section{Dr. Sanjit K. Mitra, University of California - Santa Barbara}

Sanjit Mitra is a Research Professor of Electrical \& Computer Engineering at the University of California, Santa Barbara. He has published over 700 papers in analog \& digital signal processing, digital image \& video processing, and is author or co-author of 12 books. He is a member of the US National Academy of Engineering and several foreign academies.

\section{Prof. Woon-Seng Gan, Nanyang Technological University}

Woon-Seng Gan received his BEng (1st Class Hons) and $\mathrm{PhD}$ degrees, both in Electrical and Electronic Engineering from the University of Strathclyde, UK in 1989 and 1993 respectively. He is currently an Associate Professor in the School of Electrical and Electronic Engineering in Nanyang Technological University. His research interests span a wide and related areas of adaptive signal processing, active noise control, and spatial audio. He has published more than 250 international refereed journals and conferences, and has granted seven Singapore/US/Japan patents. He had co-authored three books on Digital Signal Processors: Architectures, Implementations, and Applications (Prentice Hall, 2005), Embedded Signal Processing with the Micro Signal Architecture, (Wiley-IEEE, 2007), and Subband Adaptive Filtering: Theory and Implementation (John Wiley, 2009). He is currently a Fellow of the Audio Engineering Society(AES), a Fellow of the Institute of Engineering and Technology(IET), a Senior Member of the IEEE, and a Professional Engineer of Singapore. He is also an Associate Technical Editor of the Journal of Audio Engineering Society (JAES); Associate Editor of the IEEE Transaction on Audio, Speech, and Language Processing (ASLP); Editorial member of the Asia Pacific Signal and Information Processing Association (APSIPA) Transactions on Signal and Information Processing; and Associate Editor of the EURASIP Journal on Audio, Speech and Music Processing. He is currently sitting in the Board of Governor of APSIPA.

\section{Mr. Phyo Ko Ko, Nanyang Technological University}

PHYO KO KO received his BEng in Electrical and Electronic Engineering from Nanyang Technological University, Singapore in 2012. He is currently employed as a Project Officer in Digital Signal Processing Lab of School of Electrical and Electronic Engineering in Nanyang Technological University. His interests span in the areas of real-time audio signal processing, Java programming, C++ programming, android software development and audio modulation/demodulation.

\section{Mr. Hai Nguyen Duy, Nanyang Technological University}

Hai Nguyen Duy received his BEng in Electrical and Electronic Engineering from Nanyang Technological University, Singapore in 2014. He is currently employed as a Project Officer in Digital Signal Processing Lab of School of Electrical and Electronic Engineering in Nanyang Technological University. His interests span in the areas of real-time audio signal processing and $\mathrm{C}++$ programming. 


\title{
ON THE DEVELOPMENT OF AN OPEN-SOURCE LEARNING MANAGEMENT SYSTEM FOR ENGINEERING STUDENTS
}

\begin{abstract}
With the availability of open source learning management system (LMS) software, it is now possible to create interactive, feature rich, and customized learning materials quickly and easily. In this paper, we describe the preliminary framework of an on-line tutorial source (OnTEn) for engineering courses containing review modules that can be quickly developed using a customized Drupal CMS (Content Management System). The Drupal platform can be used for quick content organization and management, and publication of contents. The online tutorial being developed contains a series of bite-sized modules that allow the students to review through their foundation materials in flipped learning or can serve as quick reference guides. The unique feature of the OnTEn is that authors contributing the review modules need to follow standard guidelines and style templates for uniformity. The review module is submitted to a central review server and included in the website after it has been reviewed and approved for publication by a member of the Editorial Board to ensure the quality of the content. This paper reviews the OnTEn platform and describes how contributors from different universities/institutions can contribute to this effort in creating a high-standard repository of review modules for engineering students.
\end{abstract}

\section{Introduction}

This paper describes the general framework of a web-service platform, called "Online Tutor for Engineering Students" (OnTEn), which contains student-friendly, easy-to-navigate review modules for undergraduate students in engineering, that is under development. These modules aim to assist regular courses being offered by universities. One of the main goals of this website is to provide a set of review modules on topics that are taught in earlier courses. In this way, we provide a very flexible learning platform that students can use before and after the actual lecture. Each module includes a short explanation of the topic along with worked-out examples, and drill problems with answers. In addition the module has been designed to provide feedbacks on student performance for a better understanding of the relevant topic. Furthermore, this web service strives to serve as a companion platform for students to review concepts on their own time outside class.

\section{Web-Hosting Platform}

To select a platform that is optimal to host the OnTEn e-learning site, we evaluated several open source softwares available in the public domain for their suitability, as the system being developed must be easily ported and shared across different academic institutions in due course. Furthermore, it is necessary to ensure that the selected open source software has the functions for web hosting, content management, and learning management. These functions are very important to the success of the project and therefore, much effort has been spent on the initial platform feasibility study in the first phase of the development of e-learning site. 
The web-hosting platform is an operating system that the server, along with all the necessary software, runs on and is the first component one needs to consider when developing a webservice. A variety of hosting platforms are available for web hosting. For this project, we considered two of the most popular platforms, Windows and Linux. Although both platforms are capable, it was decided to use Linux platform due to its popularity, open-source nature, and having a dedicated Linux distribution for hosting server, which translates to better reliability.

\section{Content Management System}

The Content Management System (CMS) [1] is a software system that provides website authoring, collaboration, and administration tools designed to allow users with little knowledge of programming language to create and manage website content with relative ease. Such a system, which normally would take a lot of time and resource to develop, still need to be maintained and updated with new features. Hence, it is more cost and time effective to use one of the available CMS and concentrate on developing new features.

For the purpose of the project, which requires a heavily customized web-service, choosing a flexible and reliable open-source CMS supported by a strong community of developers is important. Among the vast number of CMS available, the three most popular CMS, namely, WordPress, Joomla, and Drupal were carefully examined [2]. Each CMS has its own advantages and disadvantages in terms of features, capability, flexibility and ease of use [3]. Figure 1 shows the usage statistics for the top $10 \mathrm{CMS}$ technologies.

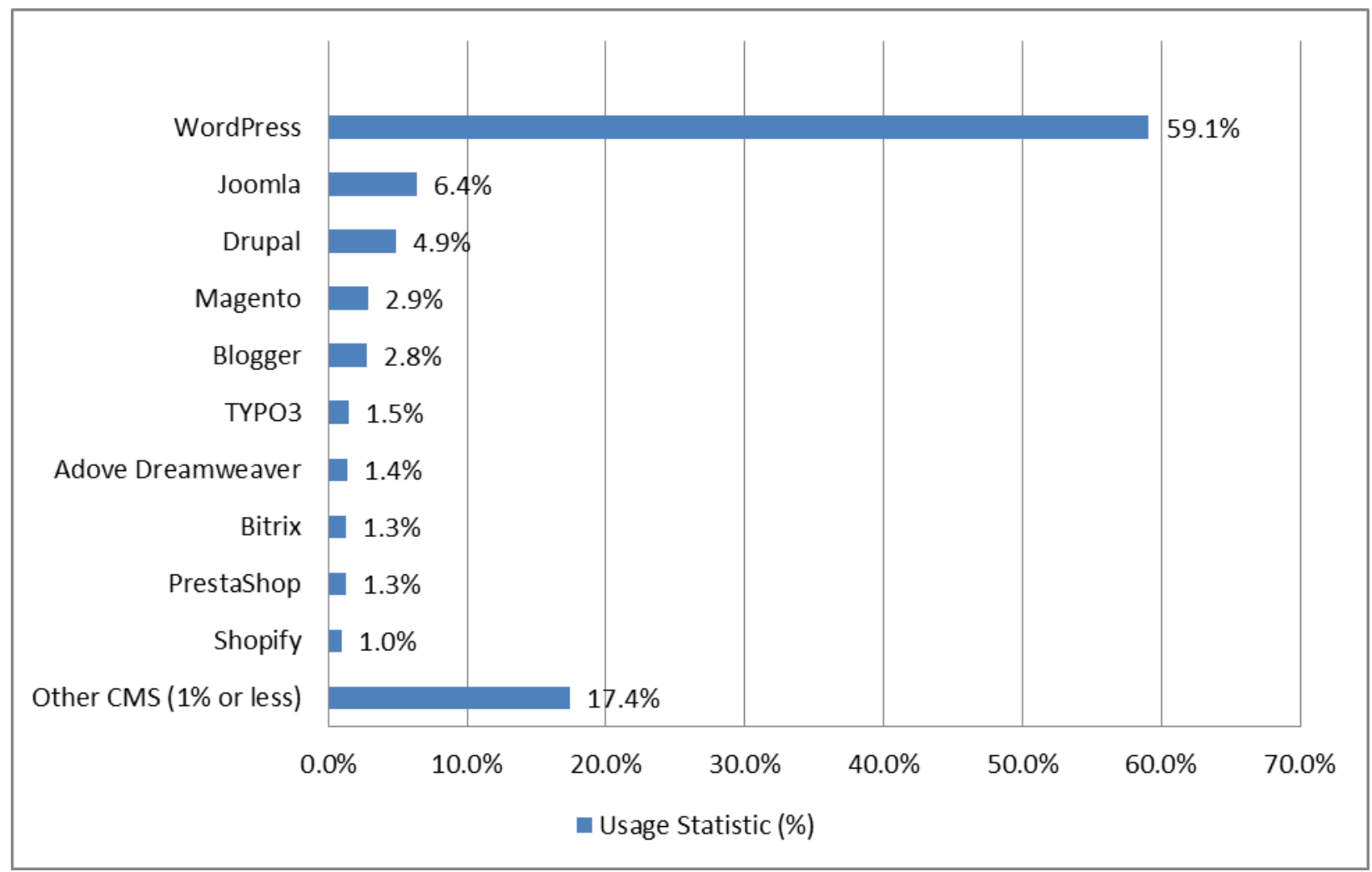

Figure 1: The usage statistics for top CMS technologies - Adapted from [2]. 
Although WordPress is the most popular CMS, it is more tailored towards blogs and new articles. However it would require extensive modifications to the framework to suit our needs. Joomla offers more flexibility than WordPress, however, it still falls short compared to Drupal in handling complex content relationships. Due to the complexity of the project, Drupal's ability to have complex relationships and access control of content provides more benefit for the development of the web-service in long term. Thus, Drupal has been chosen for its flexibility, extensibility, and having an active community contributing to the various modules.

\section{Learning Management System}

Learning Management System (LMS) is a similar type of software application, like CMS, but it is much more robust and education-focused than CMS. A notable example is the Blackboard software [4], which provides a virtual learning environment and course management system. Blackboard has been widely implemented at various universities and institutes all over the world. As the aim of the OnTEn website is to provide review-modules for engineering students, an open-source LMS has also been investigated.

Among the top LMS available [5], only Moodle [6] provides an open-source LMS allowing customization. Compared to Drupal, which can also offer an LMS experience by installing the optional Opigno [7] package, Moodle is a better full-fledged LMS that is specifically designed for course management and user interaction with a curriculum base, both by teachers and students. In terms of feature, Moodle is comparable to commercial LMS, such as Blackboard and Edmodo. However, too many features can also use up more resources and might not even be useful compared to a simple, customized Drupal site [8],[9].

In term of customizability, Moodle is built to address very specific needs in the education sector, whereas Drupal can be customized for many different applications, including education oriented websites. From the developer's point of view, one could easily build the functionality that exists in Moodle with Drupal. However, it is much harder to modify Moodle to do all the functions that Drupal can do. After consideration of all the above factors, Drupal has been selected as the platform to develop the OnTEn web-service.

\section{Website Layout and Navigation}

The layout of the OnTEn website has been designed to be simple, intuitive, and easy to use for its users. It contains two major blocks: navigation side bar and main content window, as shown in Figure 2. The main content window provides a canvas and appropriate controls for course content delivery, content modifications, commenting, reviewing, and submitting new review modules. The navigation menu handles website navigation, courses navigation, main menu navigation, and user login.

The main items of the OnTEn website are listed below:

- The 'Main menu' navigation bar links to the 'Introduction' webpage;

- 'Review Modules' list all review modules available;

- 'Advisory Board' shows the list of academic leaders, who have agreed to serve as 
advisors;

- 'Contributors' webpage lists all contributors of review modules;

- 'Editorial Board' webpage lists faculty members who have agreed to evaluate review modules submitted for possible inclusion in the website;

- 'Feedback' webpage for user feedback; and

- 'Disclaimers' webpage to delimit the scope of the website.

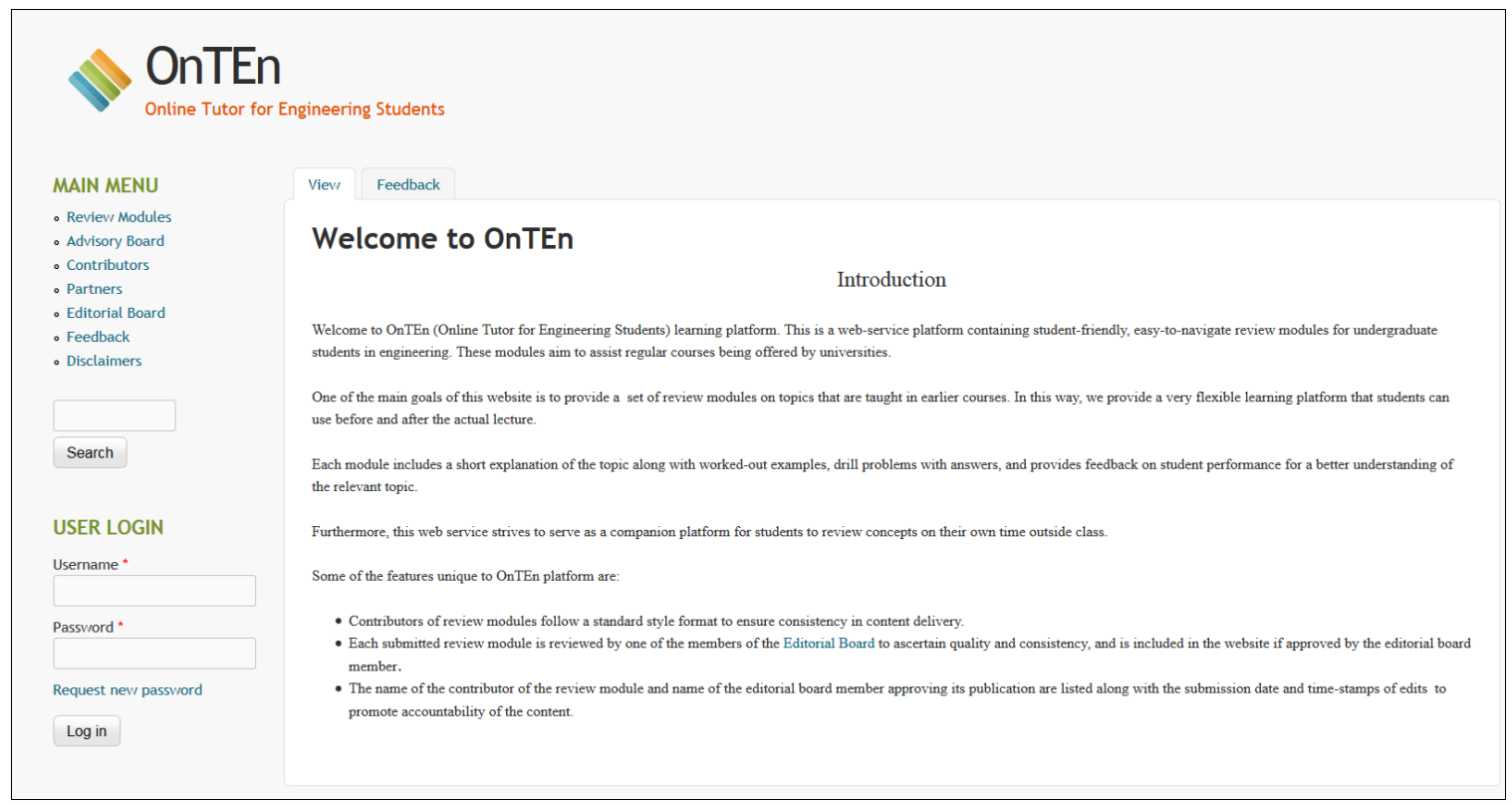

Figure 2: OnTEn website layout with navigation menu and main content window layouts.

After a review module has been submitted by a contributor, it is first checked by an appropriate member of the editorial board to ensure that it is correct and has been prepared according to the style manual for conformity with other review modules housed at the website. Every approved review module is appended with contributor's name, affiliation, date of submission, and the name of the editorial board member who reviewed the module and approved its inclusion as shown in Figure 3 to ensure its quality and accountability, and to keep track of modifications carried out on the review module based on the editorial board member's comments.

\section{Block-Diagrams}

Submitted by: Nguyen Duy Hai on 2015-06-26 12:36:19

Approved by: Phyo Ko Ko

Fields of study: Electrical \& Electronics Engineering

\section{Block-Diagrams}

Continuous-Time Systems

The input-output relation of an LTI continuous-time system is usually given in the form of a linear constant coefficient differential equation:

$$
\frac{d^{N} y(t)}{d t^{N}}+q_{N-1} \frac{d^{N-1} y(t)}{d t^{N-1}}+\cdots+q_{1} \frac{d y(t)}{d t}+q_{0} y(t)
$$

Figure 3: A review-module with its contributor, reviewer, and time-stamps shown. 


\section{LaTeX-to-HTML Converter}

The OnTEn website requires a contributor to style his/her review-module in LaTeX format using style file developed for the website. For the display of the review module on the computer screen, the LaTeX file needs to be first converted to the HTML format. To this end, we have developed a LaTeX-to-HTML conversion tool, which takes in LaTeX formatted document (.tex file) as input and produces the HTML formatted document (.html) as output. The TeX Live [10] LaTeX library and its applications, mk4ht.exe and htlatex.exe, have been used to implement the conversion from LaTeX-to-HTML format. The conversion tool also includes pre-processing and post-processing components to correct any styling errors resulting from custom commands used in the provided LaTeX styles file during the translation process.

The pre-processing component includes features, such as attaching the source LaTeX file to style file template: to conform to the style according to the template; to remove unpaired brackets in the equations, which can lead to conversion errors; to substitute custom commands found in the LaTeX source file with generic commands to overcome unknown command errors; and to create a new formatted LaTeX file to store the pre-processed source file.

The post-processing component includes features, such as embedding CSS file to the HTML output file: to satisfy one file upload requirement by Drupal; to delete the LaTeX file created by pre-processing; to resolve indentation inconsistencies resulting from the conversion; and to automatically upload the final HTML file to the OnTEn website.

A Drupal module, named Tex2Node, has been developed to serve as the front-end user interface for the LaTeX-to-HTML conversion tool. It provides a simple user interface to complete the submission process, as shown in Figure 4, which requires the contributor to name the reviewmodule, select the field-of-study where it should be listed, and choose the LaTeX source file to be uploaded. 


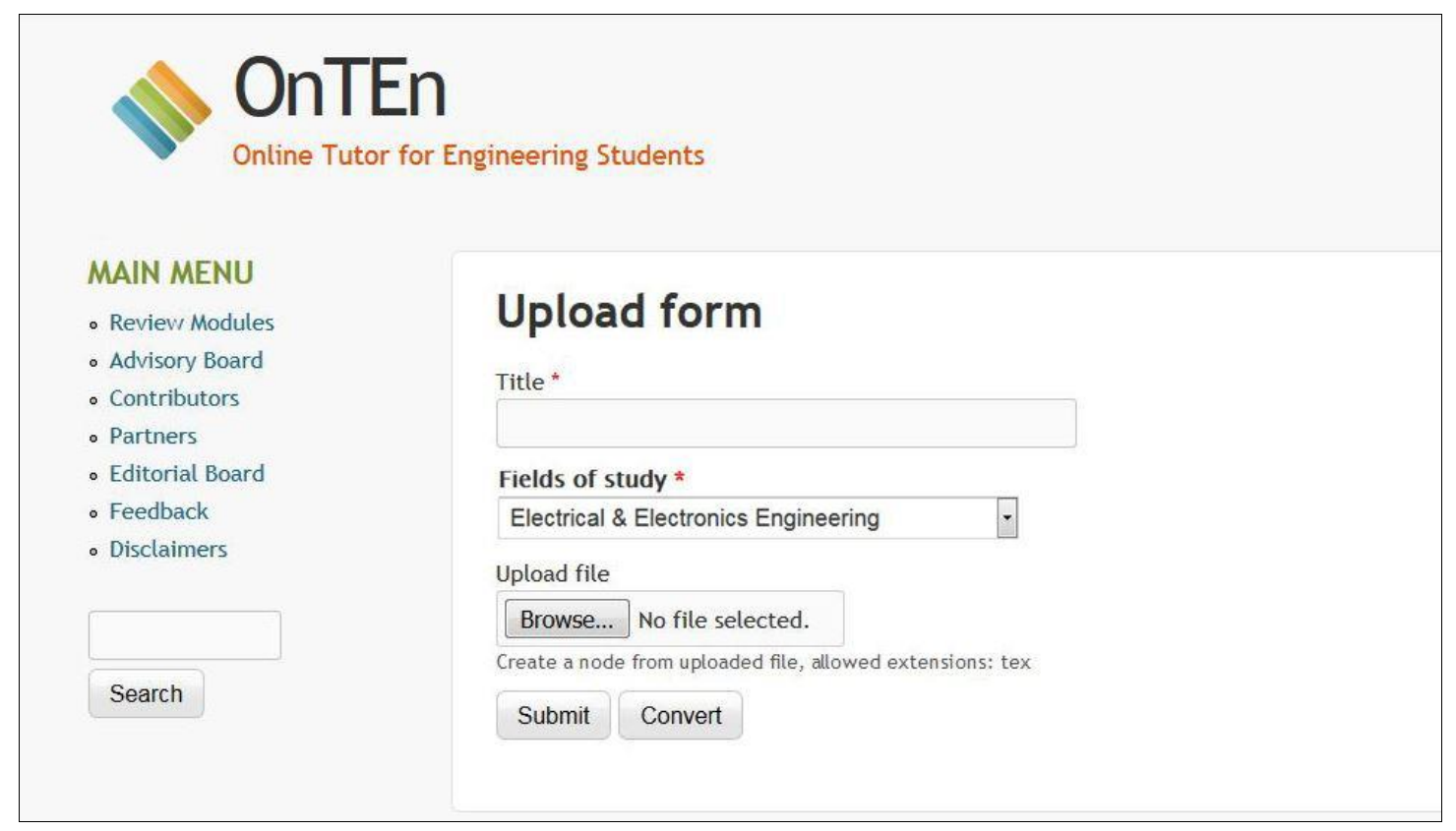

Figure 4: Tex2Node Drupal component for submitting LaTeX formatted review-modules.

\section{Submission Process}

Figure 5 shows the flow chart from the review stage to the eventual publication of the review module in the OnTEn website. Every contributor will be asked to agree to a set of "Terms and Conditions" before signing up for the website. Each field of study will have an Editorial Board specialized in that field assigned to review the content of the submitted module. The Drupalbased OnTEn system stores the previous revision of the module so that site administrator can keep track of the changes. The add-on Drupal Workbench [11] makes sure that the modules go through multi-layer of checking before being made available to students. 


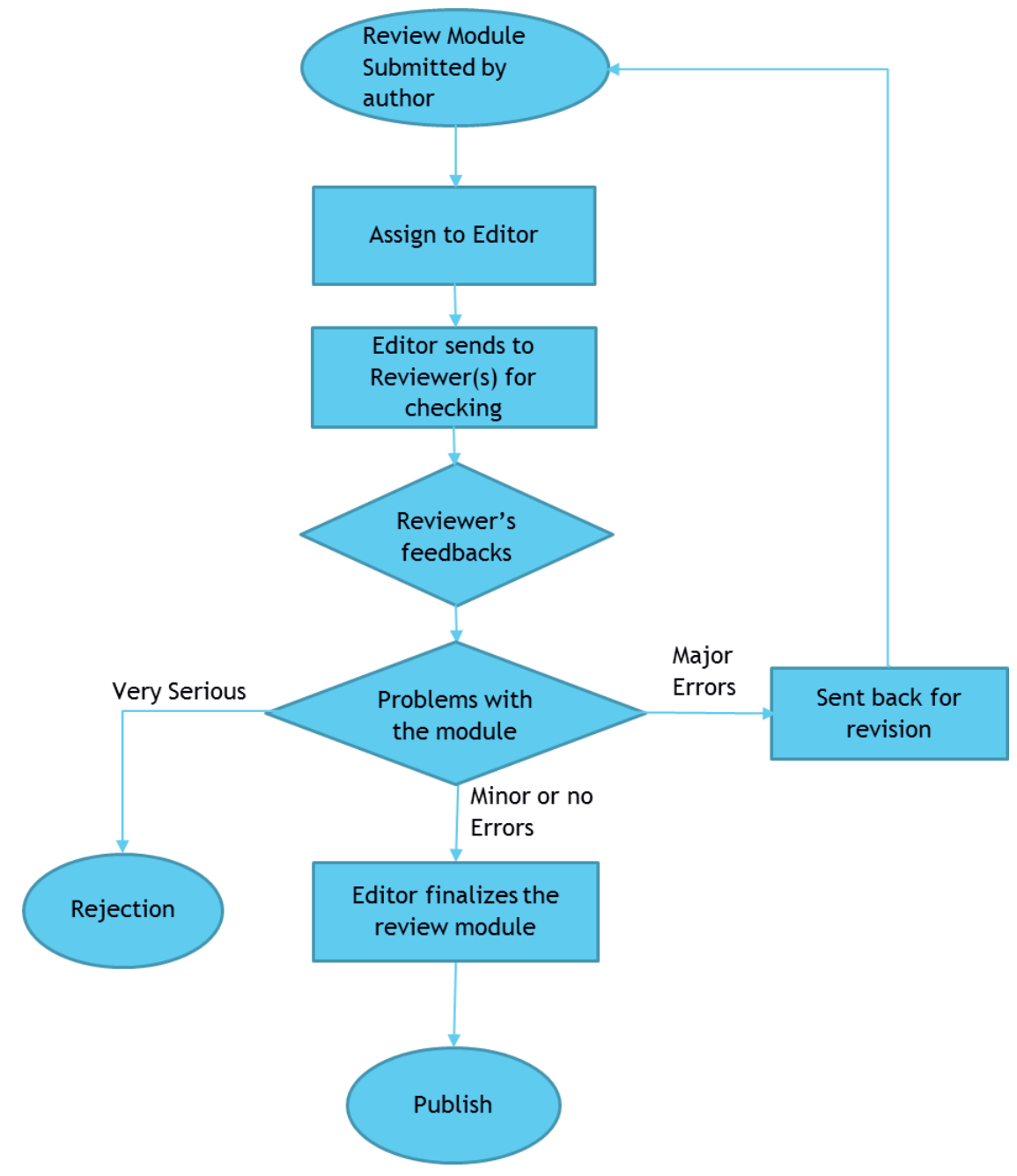

Figure 5: New module submission process.

\section{A Pilot Trial in Launching the Review Modules for ARM Programming}

A pilot trial had been conducted for the course Microprocessor (EE3002) in the School of Electrical and Electronic Engineering at the Nanyang Technological University, Singapore during the first semester of the academic year 2015-2016. A preliminary batch of 13 review modules, as shown in Figure 6, was made available to the students taking this course. These review modules contain basic fundamental concepts on how data is represented, loaded, stored, and moved within the microprocessor; how simple code can be programmed onto the microprocessor; operations of stack memory; and why and how interrupt is being carried out in the ARM microprocessor. Each review module is purposely kept to 2-3 pages in length to retain the relative short-attention span of the students, and provide links to other related review modules for students to expand their knowledge. We also recommended students to refer to a few review modules before attending lecture or a discussion session during tutorial, which can form part of a component in flipped learning classroom. This approach allows students to be familiar with some knowledge apriori and have more confidence in applying what they have just learned into answering questions or solving problems. 


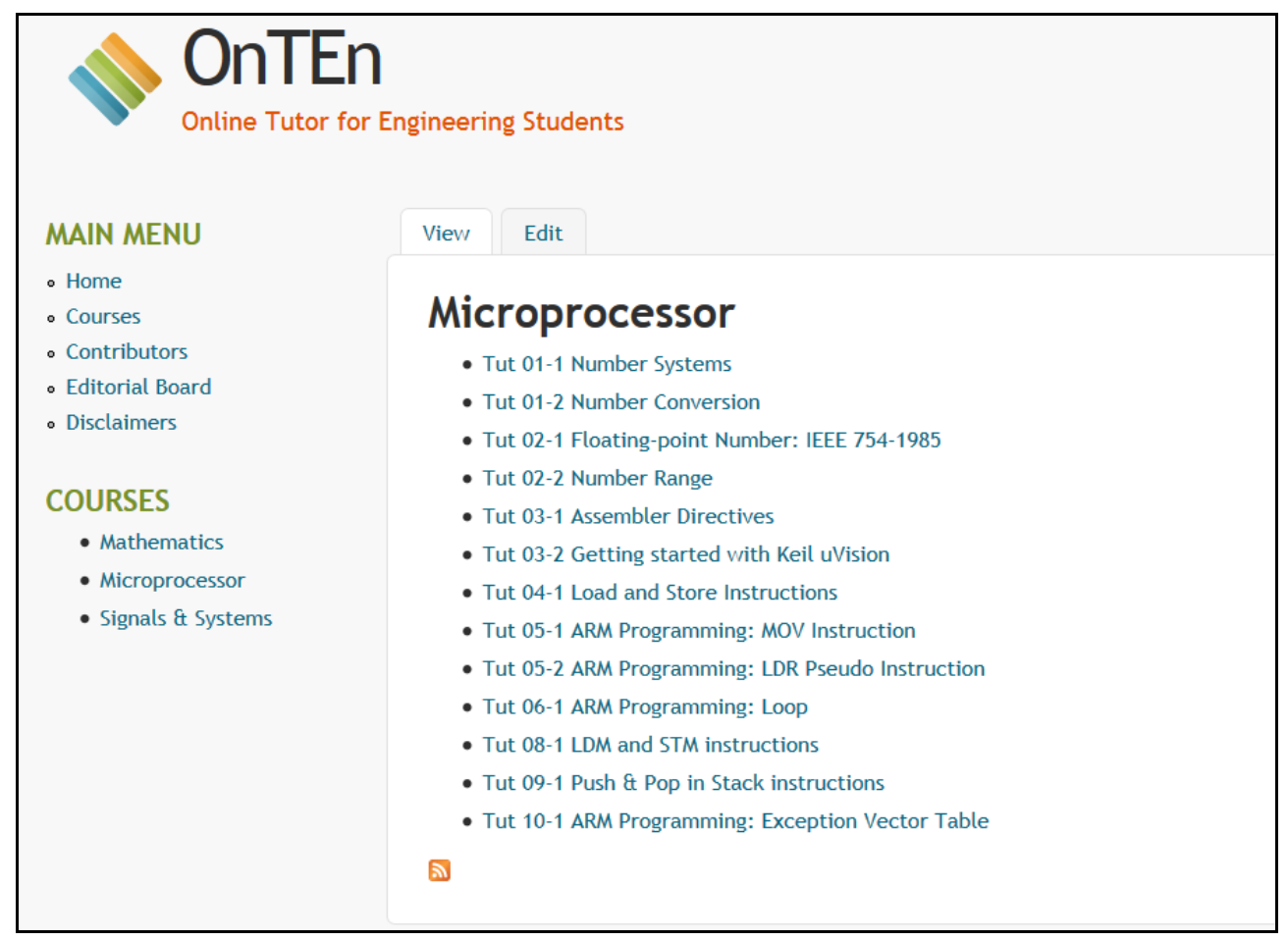

Figure 6: Review Modules for ARM Microprocessor.

These Microprocessor review modules aim to provide timely snippet of just enough information for them to understand the microprocessor architecture and start doing some programming exercises in assembly language, and gradually gaining their confidence in programming for those students who lack the background. Feedback from previous batches of students taking the Microprocessor course mainly stated the lack of knowledge in getting started. However, lecture notes and textbooks are generally organized in chapters with many topics for completeness. These topics are usually detailed in great length with little programming examples, diagrams, and some of the details may not be necessary for students to start programming or answering tutorial questions. Therefore, review modules are not meant to replace lecture notes or textbooks, but rather to provide quick information that students will need in doing their homework, and provide some pre-requisite knowledge before they can understand more advanced topics in a just-in-time and just-enough approach.

Students participated in this pilot trial also were requested to answer short survey questions, shown in Figure 7, at the end of each module. These survey forms were returned to the server, anonymously, but tagged with the module to see which modules were more commonly viewed by students and which were less frequently visited. The anonymity allowed the students to freely provide feedback. Besides the 5-grade score sheet, students were encouraged to write in their comments and suggest new review modules they would like to see. The feedback helps the module contributor to make changes and tailor the module's contents more closely to the students' need. 


The modules were easy to read and understand. *
The modules helped me understand the general concept *
The modules provide me with quick reference and allow me to work through my tutorial more
effortlessly *
I would recommend this site to friends who need help. *
Please take a moment to complete this brief survey. Your honest feedback will help improve the website so that we can make future modules more useful for your study.
Other Feedback

Figure 7: User feedback survey questions.

By the end of the trial period, there were a total of 64 feedbacks from 20 students in the class. Students were allowed to submit different feedbacks for different modules, including text comment on how useful these modules are; some were extremely helpful in spotting typos in the modules and gave some suggestions to improve on the layout and content. As feedback was optional, not all students provided feedback. But, we tracked how often a student accessed a particular module and from there, determined the need to introduce more review modules related to a particular topic, where most students need some guidance.

Feedback on this module

\begin{tabular}{|c|c|c|c|c|c|}
\hline & $\begin{array}{l}\text { STRONGLY } \\
\text { AGREE }\end{array}$ & AGREE & $\begin{array}{l}\text { NO } \\
\text { OPINION }\end{array}$ & DISAGREE & $\begin{array}{l}\text { STRONGLY } \\
\text { DISAGREE }\end{array}$ \\
\hline The modules were easy to read and understand. & 49 & 15 & 0 & 0 & 0 \\
\hline The modules helped me understand the general concept & 45 & 18 & 1 & 0 & 0 \\
\hline $\begin{array}{l}\text { The modules provide me with quick reference and allow me to } \\
\text { work through my tutorial more effortlessly }\end{array}$ & 45 & 14 & 5 & 0 & 0 \\
\hline I would recommend this site to friends who need help. & 49 & 15 & 0 & 0 & 0 \\
\hline
\end{tabular}

Figure 8: Survey Results.

\section{Future Work}

Currently, the review modules on Mathematics of Engineering and Microprocessor are being class-tested in their respective undergraduate courses at the School of Electrical and Electronic Engineering, Nanyang Technological University, Singapore.

The next phase of the development of OnTEn website is to add more engineering contents. To this end, we have visited a number of universities in several countries and plan to continue the 
visits to several other universities. The universities visited so far are the University of Southern California, Los Angeles, California (USA); University of California, Davis, California (USA); California State University, Northridge, California (USA); Chinese University of Hong Kong (Hong Kong); the Hong Kong University of Science \& Technology (Hong Kong); and the Indian Institute of Technology - Madras (India). The faculty members at each of these universities have agreed to provide review modules. Moreover, the contents of the OnTEn website will be included in the website of National Program on Technology Enhanced Learning (NPTEL), a joint venture of seven Indian Institutes of Technology (IITs) and the Indian Institute of Science (IISc), Bangalore, and funded by the Ministry of Human Resources and Development, Government of India. NPTEL provides course-wares in the form of video lectures and web courses. There are over 350 video courses, and over 12000 video lectures across all areas of engineering.

We have been invited to present a talk at the 2016 Engineers Conclave (EC2016) to be held early September this year at the Indian Institute of Technology, Madras in Chennai, India. The Engineers' Conclave is an annual conference organized by the Indian National Academy of Engineering (INAE), which provides a platform for engineers and engineer-scientists to address some of the major challenges in engineering. This year's conference is focusing on engineering education. INAE is organizing EC2016 in collaboration with Indian Institute of Technology, Madras.

At present the OnTEn website is password protected. We expect that there will be a sufficient number of contents in the website within a year. We shall then make the website available to anyone in the world. The website will also include a list of review modules that are not yet available and anyone browsing the website can submit the review module of one or more from this list. However, each such review module has to be prepared using the style manual of the website and the submitted module will be reviewed by an appropriate member of the Editorial Board. Only if the submitted module is approved, it will then be included in the OnTEn website.

\section{Concluding Remarks}

In this paper, we outlined a review module website that has been developed using an open source learning management system (LMS) software, known as Drupal. This online learning platform, called "Online Tutor for Engineering Students" (OnTEn), allows the student quick access to fundamental engineering information/definitions/knowledge in bite-sized format. The unique feature of this platform is to ensure high quality and just-enough information, coupled with examples and drill exercises for students to gain confidence in a particular topic and take ownership in their learning. Therefore, we are in the process of soliciting educators who can contribute to the review modules for different engineering topics. A preliminary trial has been conducted in the School of Electrical and Electronic Engineering at the Nanyang Technological University in Singapore, and will be extending to different courses conducted at different campuses in the coming teaching semester.

\section{Acknowledgements}


The work reported in this paper has been supported in most part by a grant from the Singapore Millennium Foundation, under the project code: M4061449, and in part by an Instructional development grant from the University of California, Santa Barbara. The authors also thank Dr. George Michaels, Executive Director, Instructional Development, University of California, Santa Barbara for his assistance in the development of the website. 


\section{References:}

[1] Drupal.org, (2016). Drupal - Open Source CMS | Drupal.org. Available at https://www.drupal.org/

[2] W3techs.com, (2016). Usage Statistics and Market Share of Content Management Systems for Websites, January 2016. Available at http://w3techs.com/technologies/overview/content_management/all

[3] Patel, S.K.; Rathod, V.R.; Parikh, S., "Joomla, Drupal and WordPress - A statistical comparison of open source CMS," in Proc. 3rd International Conference on Trends in Information Sciences and Computing (TISC), pp.182-187, December 2011

[4] Blackboard.com, (2016). Blackboard | Reimagine Education | Education Technology \& Services. Available at http://www.blackboard.com

[5] Capterra.com, (2016). Best LMS (Learning Management System) Software | 2015 Reviews of the Most Popular Systems. Available at http://www.capterra.com/learning-managementsystem-software/\#infographic

[6] Moodle.org, (2016). Moodle - Open-source learning platform |Moodle.org. Available from https://moodle.org/

[7] Opigno.org,. (2016). Opigno LMS | Learning Management done Right $\mid$ Drupal e-learning distribution. Available at https://www.opigno.org/

[8] Nicholson, K., Tokman, M., \& Maniscalco, T. (2014). Faculty Feedback on Blackboard Course Management System. Cornell University. Available at http://acadtech.cit.cornell.edu/files/2014/08/FacultyBbFinalReport-17zixco.pdf

[9] Lensyoga.com, (2016). Drupal as an LMS. Available at http://lensyoga.com/node/drupal$\operatorname{lms}$

[10] Tug.org, (2016). TeX Live - TeX Users Group. Available at https://www.tug.org/texlive/

[11] Drupal.org, (2016). Workbench $\mid$ Drupal.org. Available at https://www.drupal.org/project/workbench 